\title{
Cilio-retinal arterial circulation in central retinal vein occlusion
}

\author{
DAVID MCLEOD \\ Moorfields Eye Hospital, London
}

The presenting fundoscopic picture in clinical central retinal vein occlusion is characterized by hyperaemia of the optic disc, venous engorgement, and widespread retinal oedema and haemorrhage. Retinal oedema results from loss of the physiological integrity (or 'blood-retinal barrier' function) of capillaries and small venules, as demonstrated by dye leakage from these sites on fluorescein angiography, while the presence of haemorrhages reflects a more severe, but temporary, disruption of the anatomical continuity of the vascular endothelium.

The two main haemodynamic consequences of central vein obstruction are an increase in intraluminal pressure within the retinal microcirculation and a reduction in retinal blood flow. Little information is available, however, concerning the relative roles of such 'hydrostatic' and 'hypoxic' factors in the formation of retinal haemorrhages and oedema, although the importance of hypoxia has been emphasized in the 'combined central vascular occlusion hypothesis' (Hayreh, 1964, 1965, 197 I, 1974). Hayreh failed to produce retinal haemorrhages by experimental obstruction of the intraorbital course of the central retinal vein, while haemorrhages did result from combined occlusion of the orbital central retinal vein and artery (Hayreh, I964, 1965). The concept was thus introduced that anoxic endothelial damage secondary to central retinal artery occlusion is an additional essential factor in the pathogenesis of haemorrhages after central vein occlusions. The critical arterial obstruction might be 'partial or complete; transitory or prolonged' (Hayreh, 1971); no haemorrhages or visual loss were considered to result from 'pure' venous occlusions.

Because both the central retinal arterial circulation and the cilio-retinal arterial circulation drain into the central retinal vein, a study of central vein occlusions in patients who possess an independent retinal arterial supply from the posterior ciliary circulation has resolved some of the problems concerning the pathophysiology of the haemorrhagic and exudative sequelae of venous obstruction. The invalidity of the 'combined occlusion hypothesis' has been demonstrated.

\section{Material and methods}

Fundus photographs and intravenous fluorescein $\stackrel{ }{-}$ angiograms from 148 cases of central retinal vein occlu- $\omega$ sion were studied. Detailed assessment of the arterial $\frac{\mathbb{D}}{O}$ circulation was sometimes difficult owing to extensive $\mathbb{\mathbb { D }}$ superficial haemorrhage in the posterior pole, so followup studies were also evaluated in many cases. The photo- $\mathbb{D}$ graphs were scrutinized for the presence of a cilioretinal arterial supply, and the pattern of presenting $\vartheta$ retinal changes within and outside the area of this $G$ supply was observed.

For the purposes of this investigation, the criteria used in the diagnosis of a cilio-retinal supply were as follows:

I. An arterial vessel emerging from the edge of the optic disc, usually supplying the macula ('cilio- $\Omega$ macular artery').

2. No communication with the central retinal artery.

3. A 'loop' at the edge of the disc as the artery passes $\rightleftharpoons$ into the retina from the border tissue of Elschnig.

4. Early filling on fluorescein angiography in time with choroidal filling and before filling of the main central $\frac{0}{0}$ artery; this aids the differentiation of a cilio-retinal artery from an early retrolaminar branch of the central artery.

In the absence of morbid anatomical proof of their ciliary origin, the above criteria are an underestimate of the number of cilio-retinal vessels present as true cilio-retinal arterioles do not necessarily fulfil criteria 3 or 4 .

\section{Results}

A cilio-retinal arterial supply was shown in 26 of $\mathrm{N}$ the 148 cases; fundus photography and fluorescein angiography were performed within a week of the $\stackrel{0}{C}$ onset of symptoms in 18 of these. In a further $32 \underset{\mathscr{D}}{\mathbb{N}}$ patients, only criteria 1 and 2 (but neither criterion. 3 nor 4 ) were fulfilled; such vessels were therefore $\frac{T}{0}$ considered to represent early divisions of the $\frac{O}{\mathbb{D}}$ central artery, although it is probable that several of $\frac{\Omega}{\Phi}$ these arterioles were ciliary in origin.

In 17 patients, no significant difference could be discerned in either fluorescein perfusion or in the 8 distribution of pathological features (in particular 


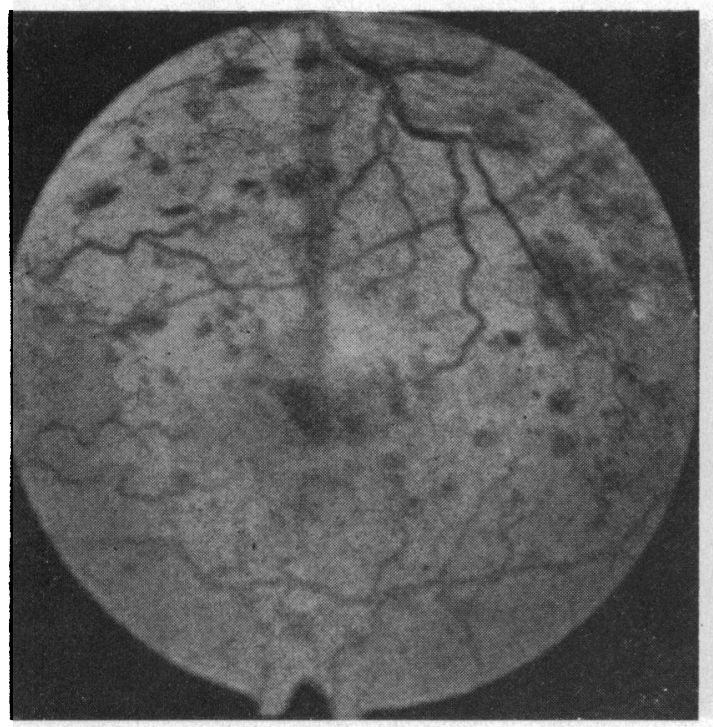

( $\mathrm{I} a)$

FIG. I Case I on presentation

(a) Fundus photograph of macula from colour transparency : retinal oedema and scattered haemorrhages

(b) and (c) Fluorescein angiogram of disc and macula

(b) Early arterial phase of central arterial transit; capillary phase of cilio-retinal arterial transit

(c) Venous phase: venous distension and retino-ciliary bypass channel on disc; fluorescein leakage into macula (especially where cilio-retinal circulation contributes to perifoveal capillary arcade (arrow))

haemorrhages) between the capillary beds supplied by the central retinal and cilio-retinal arterial circulations (bearing in mind the variable pattern of haemorrhages in the posterior pole and their often preferential peripapillary localization after central vein occlusion). 'Pre-retinal arterial' fluorescein filling of the cilio-retinal vessels was demonstrated in seven patients; in ten patients, the choroid, cilio-retinal arterioles, and central retinal arterioles all contained dye in the first available frame of the angiographic transit, but the cilio-retinal vessels showed a characteristic 'loop'. Case I was typical of this group of patients with homogeneous retinal changes; the mild presenting haemorrhagic retinopathy permitted

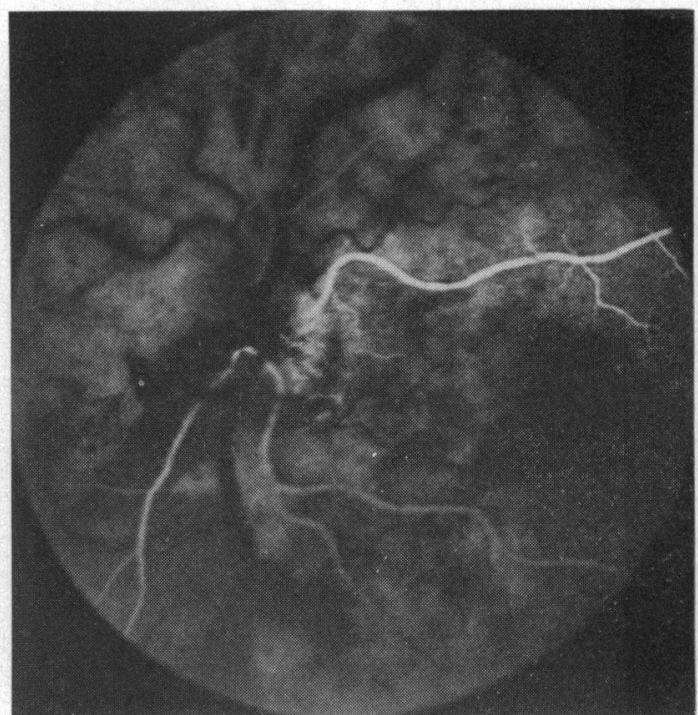

( $(b)$

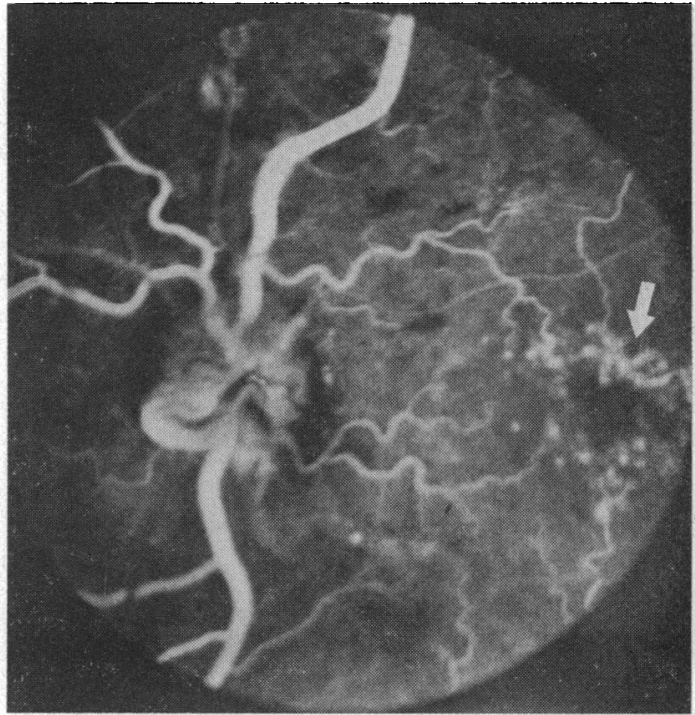

$(\mathrm{I} c)$

unequivocal demonstration of the cilio-retinal supply, and the subsequent distribution of haemorrhages and oedema also showed no predilection for one or other arterial territory.

Case I, a 44-year-old mildly hypertensive man, presented with a 2-day history of visual deterioration in the left eye (visual acuity, 6/12). The right eye had been enucleated after an accident 30 years earlier. Fundoscopy revealed a hyperaemic disc, widespread retinal haemorrhages, gross venous tortuosity, and a retino-ciliary bypass channel on the disc. Fluorescein angiography confirmed the venous outflow obstruction; the cilio-retinal supply to the superior macular area did 


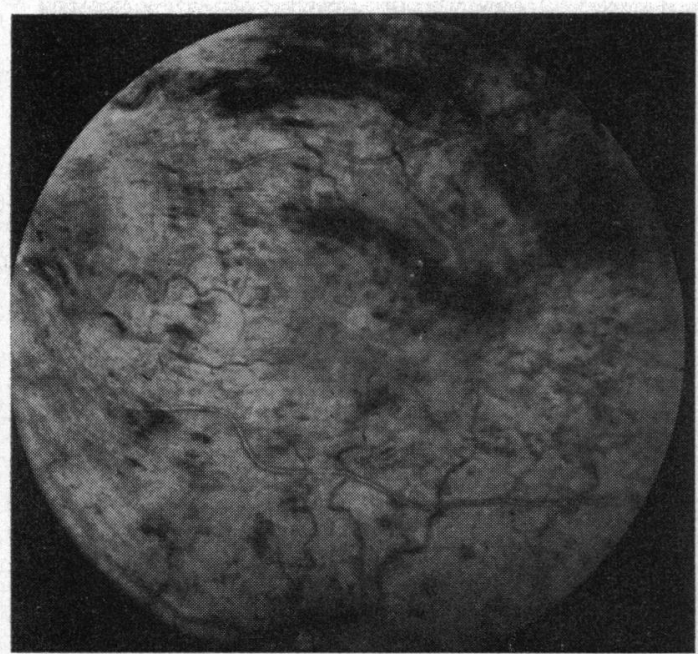

(2a)

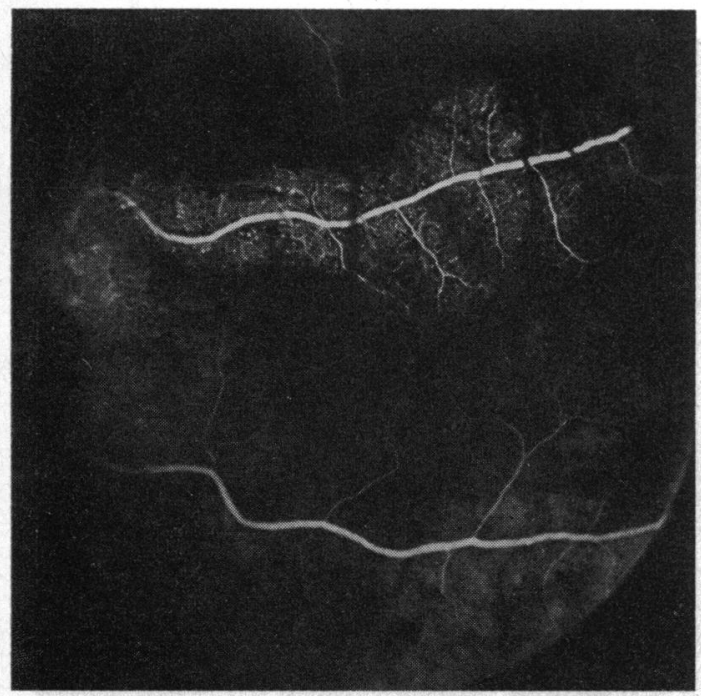

(2b)

not influence the distribution of haemorrhages (Fig. r).

No treatment was given; vision in the left eye deteriorated over the next few weeks to $6 / 18$ with further haemorrhages, macular oedema, and 'coarsening' of the capillary pattern in the posterior pole (Fig. 2). However, the haemorrhages and oedema eventually cleared and vision improved to 6/9.

In several other patients, however, angiography provided evidence of a substantial difference in perfusion in the cilio-retinal circulation compared with that in the central arterial territory-for example, Case 2.

Case 2, a 72-year-old woman, under treatment for hypertension presented with acute visual loss in the

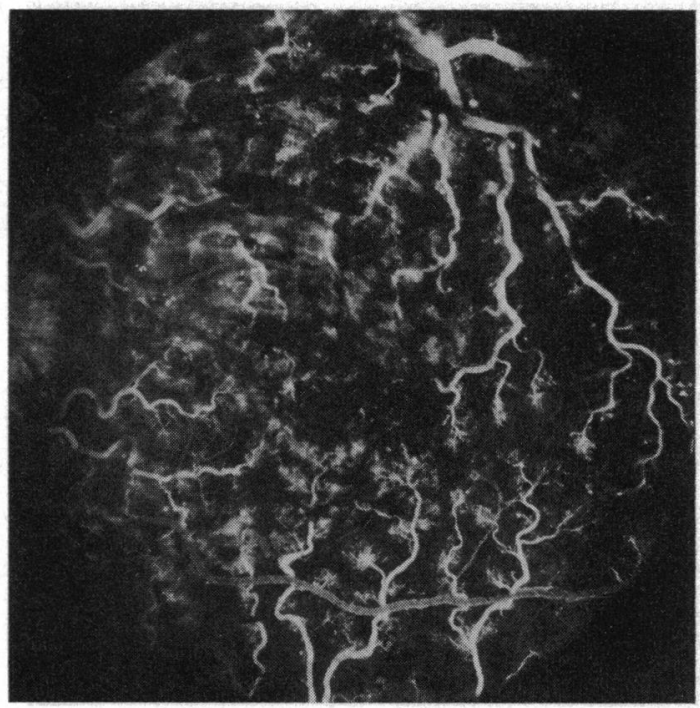

(2c)

FIG. 2 Case : two months after presentation

(a) Fundus photograph of disc and macula from colour transparency; venous engorgement, oedema, and haemorrhages

(b) and (c) Fluorescein angiogram

(b) Arterial phase of central arterial transit; capillary phase of cilio-retinal arterial transit showing microaneurysm formation

(c) Venous phase: fluorescein leakage from small venules

right eye of 24 hours' duration (visual acuity, 6/60); vision in the left eye had failed 6 weeks earlier because of a severe central vein obstruction with extensive capillary closure. Fundoscopy on the right showed tortuosity of the veins, scattered discrete haemorrhages, and yellowish deep retinal oedema above and nasal to the fovea (Fig. 3).

A double cilio-macular arterial supply was demonstrated angiographically. Comparison of arteriolar dye densities during the angiographic transit indicated that the cilio-retinal arterial inflow relatively exceeded (as well as preceded) that in the central arterioles, and the fluorescein transit time through the cilio-retinal circulation was shorter than that of the central retinal supply to the macula.

On presentation, the distribution of deep retinal oedema and focal fluorescein leakage from small venules closely correlated with the cilio-retinal arterial territory, and there was an excess of haemorrhages and capillary 


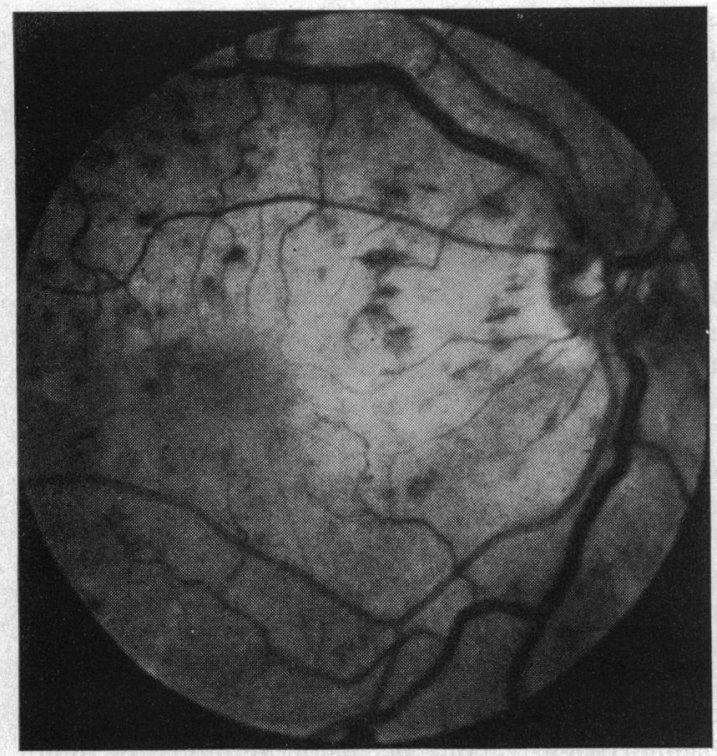

(3a)

FIG. 3 Case 2 on presentation

(a) Fundus photograph from colour transparency : venous distension, scattered haemorrhages, and retinal oedema above and nasal to fovea

(b) and (c) Fluorescein angiogram

(b) Early venous phase of dye transit showing capillary dilatation in cilio-retinal territories

(c) Late phase: focal leakage of dye from venules in cilio-retinal arterial territories

dilatation within this area compared with an equivalent area of central arterial circulation.

Treatment with streptokinase and anticoagulants was instituted (Kohner, Hamilton, Bulpitt, and Dollery, 1974). Within 6 weeks, the visual acuity in the right eye improved to $6 / 12$, with reversal of venous engorgement and absorption of oedema and haemorrhages. Fluorescein studies confirmed the improvement in venous outflow. Secondary changes in arteriolar calibre were localized to the cilio-retinal vessels.

In seven patients with central retinal vein occlusion, retinal opacification occurred in the cilioretinal arterial territory-for example, Case 3 .

Case 3, a 50-year-old man, under treatment for angina presented with a 5-day history of hazy vision in the right eye (visual acuity, 6/60); visual field examination showed an extensive scotoma just above fixation.

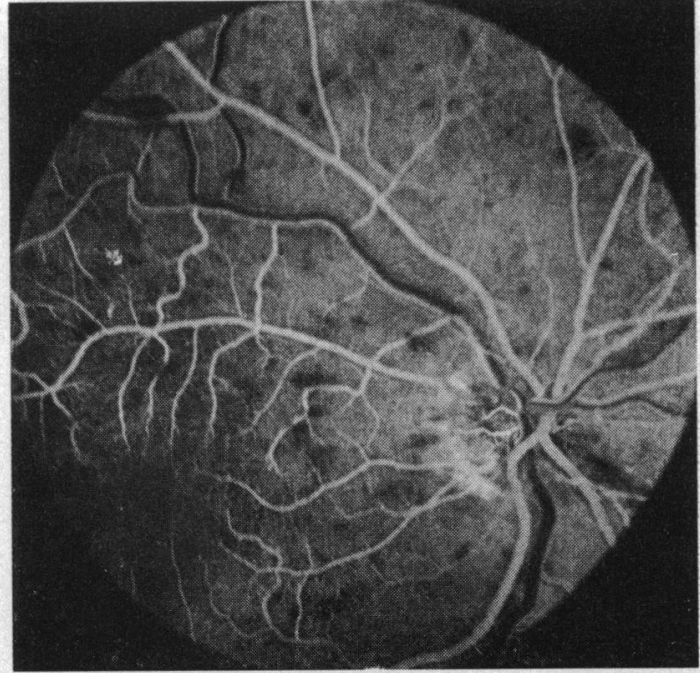

$\left(3^{b}\right)$

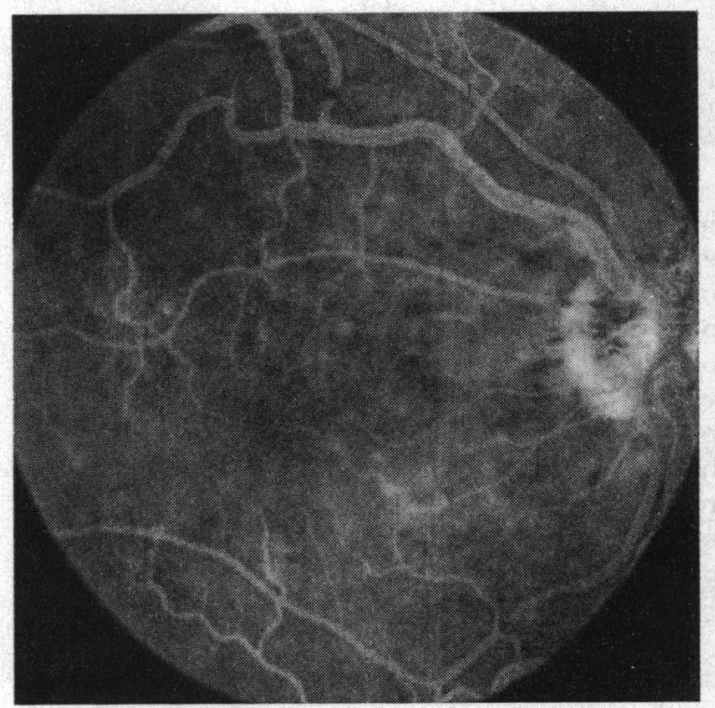

$(3 c)$

Fundoscopy revealed a swollen optic disc, venous engorgement, and widespread retinal haemorrhagesespecially in the immediate peripapillary region. A patch of white retinal opacification corresponded with the distribution of a large cilio-retinal arterial supply to the inferior macular region (Fig. 4). Fluorescein angiography confirmed the marked circulatory stasis especially in the cilio-retinal capillary bed.

During the next few weeks, the vision improved to $6 / 12$, although the scotoma was unchanged; the retinal opacification and haemorrhages cleared, and retinociliary bypass channels became evident on the optic disc.

Severely reduced capillary perfusion by fluorescein (and corresponding absolute visual field defects) 
indicated that the retinal pallor was caused by cloudy swelling of the inner retina in the distribution of the cilio-retinal arterial supply in all seven patients. In some cases, retinal haemorrhages were observed within the area of infarction (particularly when venules draining the central arterial capillary bed traversed the ischaemic cilio-retinal territory); however, compared with the surrounding central retinal circulation, the cilio-retinal infarcts were usually relatively deficient in haemorrhages on presentation-for example, Case 3-and at subsequent examinations.

Another patient presented with retinal infarction in the cilio-retinal territory, spontaneous pulsation of the cilio-retinal arterioles but few fundoscopic signs of central venous occlusion (although fluorescein studies demonstrated the central vein obstruction). Anterior chamber paracentesis resulted in haemorrhage formation on and around the optic disc and in the peripheral retina, but sparing the cilio-retinal territory (Fig. 5).

\section{Discussion}

This study was designed to put into perspective the role of arterial obstruction in the pathogenesis of the presenting fundus changes in central retinal vein occlusion. The desirability of a clinical investigation follows from the difficulty in reproducing the human condition exactly by experiment, and the pitfalls of comparing acute vascular occlu-

sions in young healthy animals with more chronic occlusions in patients with systemic vascular disease; the capillary beds in these two groups may differ considerably in their capacity to withstand increases in intraluminal pressure due to changes in microcirculatory structure that occur with age (Cogan and Kuwabara, 1963).

In the "combined central vascular occlusion hypothesis', a transient occlusion of the central retinal artery was considered responsible both for the occurrence of central vein thrombosis (via circulatory stasis) and the development of an 'ischaemic capillaropathy' essential to the formation of retinal haemorrhages when the arterial circulation is restored (Hayreh, 1971). A corollary of the hypothesis demands that, when such a pathogenic sequence operates, the area of retina with an independent posterior ciliary supply will be free of haemorrhages, since 'pure venous occlusion' conditions prevail in this territory. The identification of any instances of cilio-retinal 'sparing' in a haemorrhagic venous occlusion retinopathy might thus provide crucial evidence in favour of this hypothesis; the fact that none was evident in this study or in the literature is a strong indication of its irrelevance. Central retinal arterial occlusion, however transient, cannot be considered an essential predeterminant of haemorrhage formation after venous occlusion unless coincident transient ciliary occlusion or transient ophthalmic artery occlusion is postulated in all cases; it is equally unlikely that the haemorrhages antedated the occlusion or spread to the area of cilio-retinal supply from the surrounding circulation.

Fluorescein angiography in Case 2 provided evidence of partial stenosis of the central retinal artery and indicated that the input arterial pressure in the cilio-retinal vessels exceeded that in the central artery. The cilio-retinal capillary bed thus enjoyed a relatively greater perfusion (but was subjected to a higher mean intraluminal pressure) than the central retinal microcirculation. The resultant excess of haemorrhages and oedema in the cilio-retinal territory has several important implications:

I. 'Hydrostatic' factors are relatively more important than 'hypoxic' factors in determining the formation of oedema and haemorrhages after venous occlusions.

2. The distribution of haemorrhages is contrary to that predicted from the 'combined occlusion hypothesis'.

3. Although central retinal artery stenosis may be of significance in the genesis of some venous occlusions (Klein and Olwin, 1956; Hayreh, 1965, I97I), it paradoxically affords a degree of 'protection' to the retina from the haemorrhagic 


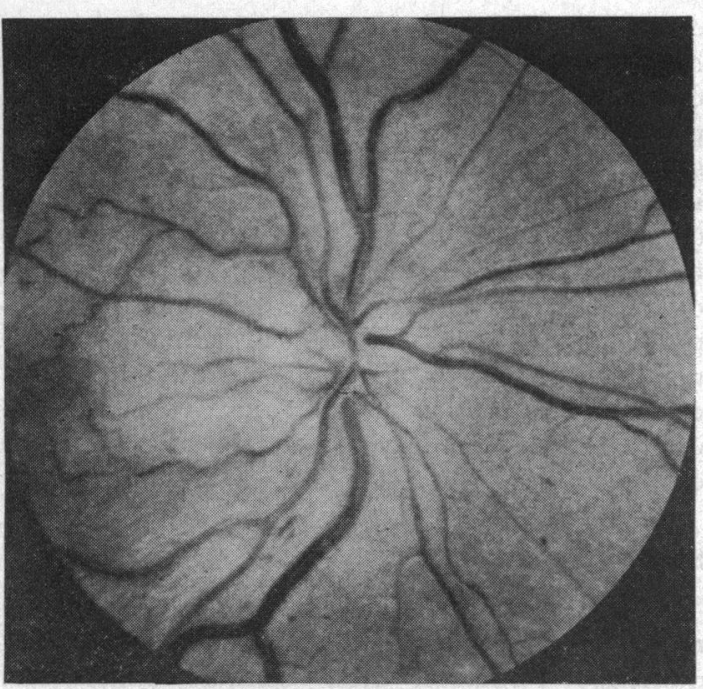

$(5 a)$

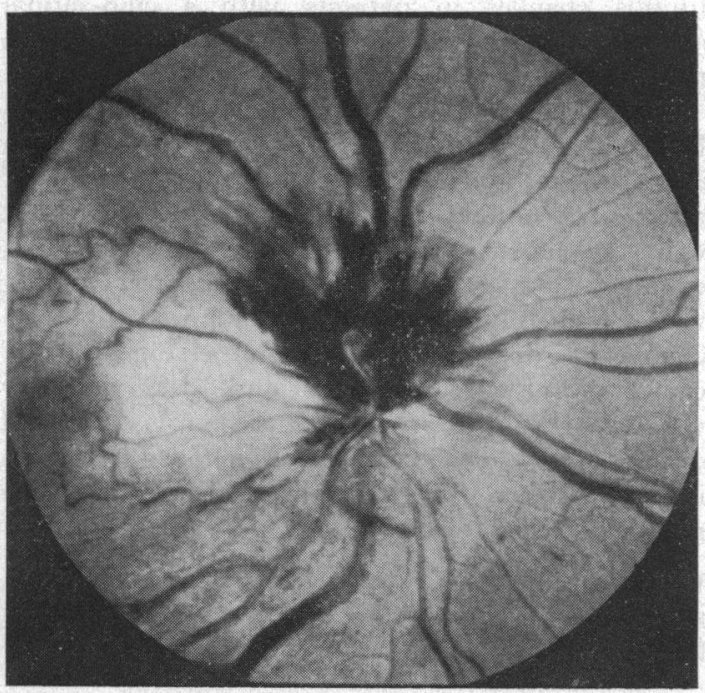

$\left(5^{b}\right)$

FIG. 5 Fundus photographs from colour transparencies

(a) Retinal cloudy swelling in cilio-retinal territories, punctate haemorrhages, and venous distension

(b) After paracentesis: increased distension and tortuosity of veins, with disc and peripapillary haemorrhages (sparing the cilio-retinal arterial territories)

and exudative sequelae of the occlusion by limiting the increase in intraluminal pressure within the microcirculation. Wise, Dollery, and Henkind (1971) offered a similar explanation for the paucity of retinal haemorrhages after central retinal vein occlusion in patients with carotid artery stenosis (the so-called 'venous stasis retinopathy' of Kearns and Hollenhorst (1963)); however, capillary perfusion is doubly compromised in these instances.

The association of central retinal vein obstruction with ischaemic cloudy swelling in the cilioretinal arterial territory will be discussed in detail in a subsequent report (McLeod and Ring, 1975); the failure of cilio-retinal capillary perfusion reflected the combined influence of partial ciliary arterial obstruction and central retinal venous occlusion. Hayreh (1971) described a patient with central vein occlusion in whom retinal haemorrhages were localized to such an area of cilio-retinal infarction; the case was submitted as clinical proof of the validity of the 'combined occlusion hypothesis'. In this series, however, a relative paucity of haemorrhages generally occurred in the ischaemic cilio-retinal territory. The lack of haemorrhages within the retinal infarct could not be ascribed to complete failure of vascular perfusion since angiography demonstrated slow capillary filling in most cases; no haemorrhages developed when cilioretinal perfusion subsequently improved (although this improvement was usually associated with a reduction in central venous outflow resistance).

In this series, cilio-retinal perfusion was insufficient to maintain neuronal viability, yєt most capillaries within the infarct withstood intraluminal pressures in excess of those encountered in 'pure arterial occlusions' (where haemorrhages are characteristically lacking). Furthermore, in comparison with the central arterial territory, the relatively low intraluminal pressures in the cilio-retinal microcirculation more than offset the potential influence of hypoxic endothelial damage in determining haemorrhage formation. It is possible that cloudy retinal swelling per se might reduce capillary transmural hydrostatic pressure gradients within an infarct; nevertheless, the singular significance of excessive transmural pressure gradients in the causation of haemorrhages was illustrated by the effect of paracentesis (Fig. 5). These observations are in keeping with electron microscopic changes in experimental focal arterial occlusions; Shakib and Ashton (1966) concluded that the ultrastructural integrity of the retinal vascular endothelium is more resistant to hypoxia than that of neural tissues. Our findings cannot be reconciled with the 'combined occlusion hypothesis', particularly as it has been applied to the role of 'prolonged partial arterial stenosis' in the pathogenesis of retinal haemorrhages after venous occlusions (Hayreh, I97 I).

That is not to deny the potential role of anoxic vascular damage in the pathogenesis of haemorrhages after venous occlusions, particularly after 
severe obstructions. In this series, haemorrhages sometimes occurred within the area of infarction (especially temporal to the macula) because of retrograde filling of the cilio-retinal capillary bed from venules draining the central arterial territory. The distribution of haemorrhages in Hayreh's Case 5 (1971) may similarly reflect the fact that large venules (with a high intraluminal pressure) traversed the parapapillary infarct.

Neither Hayreh (1964, 1965), nor Fujino, Curtin, and Norton (1969), observed a typical haemorrhagic venous occlusion retinopathy following obstruction of the central retinal vein after its emergence from the optic nerve dural sheath in monkeys. Indeed, there is only one instance of experimental central vein occlusion in primates that closely resembles the clinical condition; an incomplete neoprene latex occlusion of the central vein in a single Owl monkey resulted in venous engorgement and retinal haemorrhages, but no conspicuous disruption of retinal architecture (Fujino, and others, 1969). Thus, a distinction between fundus changes without haemorrhages and with haemorrhages is arbitary; only the degree of increase in venous outflow resistance is reflected, not a fundamental difference in pathogenesis. The limited effects of orbital central retinal vein occlusion result in part from collateral drainage communications of the intravaginal central vein with optic nerve pial veins; such collaterals are not available in clinical venous occlusions at the lamina cribrosa.

The formation of retinal haemorrhages after combined diathermy occlusion of the orbital central retinal artery and vein (Hayreh, 1965) reflected the restoration of arterial flow to the infarcted retina via optic nerve pial collaterals of the central artery, and the failure of the microcirculation (having suffered anoxic damage) to resist transmural pressure gradients which it would otherwise ordinarily have sustained. A necrotic haemorrhagic retinopathy was also produced by completely obstructing the central retinal vein (and potential retino-ciliary bypass channels) by retrograde injection of neoprene latex, which solidified in the main venous tributaries at the optic disc (Fujino and others, 1969). Owing to the gross failure of retinal vascular perfusion (with infarction of the entire inner retina), neither experiment reproduced the common clinical situation where maintenance of circulation is attributable to incomplete obstruction of the central venous lumen or to the availability of retino-ciliary bypass channels.

\section{Summary}

The hypothesis that an occlusion of the central retinal artery is an essential prerequisite for haemorrhage formation after central retinal vein obstruction has been investigated by examining the fundus changes in patients with a cilio-retinal arterial circulation; the findings are at variance with the 'combined occlusion hypothesis'.

Comparisons were made between the pathological features in two retinal capillary beds with independent sources of arterial supply-namely, the central retinal and cilio-retinal arteries-but with an obstructed venous drainage channel common to both-namely, the central retinal vein. The importance of intraluminal pressure changes (as distinct from perfusion changes) in the causation of haemorrhages and oedema after venous occlusion is stressed, and the role of arterial disease in the pathogenesis of venous occlusions is distinguished from its role in determining the sequelae of such occlusions.

I am grateful to Dr E. M. Kohner, Mr A. C. Bird, and $\mathrm{Mr} M$. D. Sanders for permission to study their patients, and to $\mathrm{Mr} \mathrm{K}$. Shemi for the illustrations.

\section{References}

Cogan, D. G., and KuWABara, T. (1963) Trans. ophthal. Soc. U.K., 83, 465

Fujino, T., CURTin, v. T., and Norton, E. w. D. (1969) Arch. Ophthal. (Chicago), 81, 395

HAYREH, S. S. (1964) Trans. ophthal. Soc. U.K., 84, 586

(1965) Brit. F. Ophthal., 49, 626

(1971) Amer. F. Ophthal., 72, 998

(r974) Symposium on 'Pathogenesis and Prevention of Retinal Vein Occlusion', Paris, (May 1974).

Junk, The Hague (in press)

KEARNS, T. P., and hollenhorst, R. W. (1963) Proc. Mayo Clin., 38, 304

KLEIN, B. A., and olwin, J. H. (1956) Arch. Ophthal. (Chicago), 56, 207

KOHNER, E. M., HAMILTON, A. M., BULPITT, C. J., and Dollery, c. T. (1974) Trans. ophthal. Soc. U.K., 94, 599

MCLEOD, D., and RING, C. P. (1975) In preparation

SHAKIB, M., and ASHTON, N. (1966) Brit. $\mathcal{Y}$. Ophthal., 50, 325

WISE, G. N., DOLLERY, C. T., and HENKIND, P. (I97I) In 'The Retinal Circulation', Harper \& Row, New York 